\title{
Marcel MAUSS
}

\section{« Divisions et proportions des divisions de la sociologie »}

Extrait de « Divisions et proportions des divisions de la sociologie » (1927, Année sociologique, nouvelle série, 2).

\begin{abstract}
Un document produit en version numérique par Jean-Marie Tremblay, professeur de sociologie au Cégep de Chicoutimi

Courriel: jmt_sociologue@videotron.ca

Site web: http://pages.infinit.net/sociojmt
\end{abstract}

Dans le cadre de la collection: "Les classiques des sciences sociales"

Site web: http://www.uqac.uquebec.ca/zone30/Classiques_des_sciences_sociales/index.html

Une collection développée en collaboration avec la Bibliothèque

Paul-Émile-Boulet de l'Université du Québec à Chicoutimi

Site web: http://bibliotheque.uqac.uquebec.ca/index.htm 
Cette édition électronique a été réalisée par Jean-Marie Tremblay, professeur de sociologie au Cégep de Chicoutimi à partir de :

Extrait de «Divisions et proportions des divisions de la sociologie » (1927, Année sociologique, nouvelle série, 2).

Polices de caractères utilisée :

Pour le texte: Times, 12 points.

Pour les citations : Times 10 points.

Pour les notes de bas de page : Times, 10 points.

Édition électronique réalisée avec le traitement de textes Microsoft Word 2001 pour Macintosh.

Mise en page sur papier format LETTRE (US letter), 8.5' x 11'”)

Édition complétée le 16 février 2002 à Chicoutimi, Québec.

\section{Fait avec}

Macintosh 


\section{Table des matières}

\section{Division concrète de la sociologie (1927)}

Extrait de «Divisions et proportions des divisions de la sociologie » (1927, Année sociologique, nouvelle série, 2).

\section{I. $\quad$ Principe}

Contenu de la morphologie sociale Contenu de la physiologie sociale

II. Avantages de cette division

III. Emploi simultané des deux méthodes de division.

IV. Utilité de cette division pour une sociologie générale concrète

V. Place de la sociologie appliquée ou politique.

$\mathrm{La}$ «sociologie de la politique », partie de la « sociologie générale » $\underline{\text { Sociologie et politique }}$ 


\section{Division concrète de la sociologie : (1927)}

$\underline{\text { Retour à la table des matières }}$

\section{PRINCIPE}

En fait, il n'y a dans une société que deux choses : le groupe qui la forme, d'ordinaire sur un sol déterminé, d'une part; les représentations et les mouvements de ce groupe, d'autre part. C'est-à-dire qu'il n'y a, d'un côté, que des phénomènes matériels : des nombres déterminés d'individus de tel et tel âge, à tel instant et à tel endroit; et, d'un autre côté, parmi les idées et les actions de ces hommes communes en ces hommes, celles qui sont, en même temps, l'effet de leur vie en commun. Et il n'y a rien d'autre. Au premier phénomène, le groupe et les choses, correspond la morphologie, étude des structures matérielles ${ }^{2}$; au deuxième phénomène correspond la

1 Extrait de «Divisions et proportions des divisions de la sociologie » (1927, Année sociologique, nouvelle série, 2).

2 Sur la notion de structure : Nous nous excusons de continuer à nous servir du mot « structure ». Il désigne en effet trois choses distinctes : 1. des structures sociales qui sont vraiment matérielles : répartition de la population à la surface du sol, à des points d'eau, dans des villes et des maisons ou le long des routes, etc.; répartition d'une société entre sexes, âges, etc.; puis d'autres choses, matérielles encore, mais déjà morales qui méritent encore le nom de structure puisqu'elles se manifestent de façon permanente, en des endroits déterminés : emplacements d'industries; groupes secondaires isolés, par exemple, dans une société composite : ainsi les quartiers nègre, chinois, italien, d'une grande ville américaine; 2 . nous appelons encore structures des sous-groupes dont l'unité est surtout morale, bien qu'elle se traduise en général par des habitats uniques, des agglomérations précises, plus ou moins durables : par exemple le groupe domestique et, à titre d'illustration : la grande famille, le groupe des parsonniers; les clans qui déjà ne sont plus constamment isolés les uns des autres et ne sont pas toujours groupés en quartiers ou en localités; 3. enfin nous appelons structure sociale quelque chose qui n'a plus rien de matériel, la constitution de la société ellemême, la constitution des sous-groupes; par exemple : un pouvoir souverain, une chefferie dans la tribu, le clan ou la famille ; les classes d'âges, l'organisation militaire, etc., tous phénomènes presque purement physiologiques, juridiques même presque exclusivement. Nous aurions voulu faire disparaître cette confusion entre faits de morphologie et faits de physiologie dans notre propre nomenclature. Nous avons essayé de réserver à ce dernier groupe de faits, rassemblements purement moraux, le nom de constitutions. Seulement ce mot ne marque pas que, tout de même, en ces faits, il y a autre chose que le droit. Par exemple, les compagnies d'un régiment, les archers ou les frondeurs d'une tribu, ont une place dans une ligne de bataille. Nous nous efforcerons 
physiologie sociale, c'est-à-dire l'étude de ces structures en mouvement, c'est-à-dire leurs fonctions et le fonctionnement de ces fonctions. Durkheim a divisé celle-ci avec précision en physiologie des pratiques et physiologie des représentations collectives.

Tandis que ceci n'est pas sûr de la division que nous suivons d'ordinaire, celle des sociologies spéciales, cette division est sans doute complète. Elle risque aussi d'être exacte, car elle est profondément concrète. Elle ne divise rien qui ne soit évidemment divisé. Enfin elle laisse tout en l'état.

Elle suit en principe les divisions de la biologie et de la psychologie.

Cependant il ne faut pas pousser trop loin cette imitation de la biologie, où d'ailleurs la distinction tranchée entre morphologistes et physiologistes, n'est pas ellemême sans danger. Ces emprunts de méthodes, de science à science, doivent être faits avec prudence. Pour nous instruire, souvenons-nous de l'erreur absurde de Comte et comment il prenait à la mécanique sa distinction de la statique et de la dynamique sociales. Et voyons les choses sous les mots. Car nous nous servons de termes que Durkheim empruntait il y a trente ans à des sciences qui ont progressé depuis et ces termes doivent être définis. La division primaire : morphologie, physiologie, doit être dégagée de tout souvenir des sciences de la vie. Ces mots même ne peuvent avoir le même sens en sociologie que dans d'autres sciences. Il faut préciser celui que nous leur donnons. Nous accentuerons ainsi la ligne générale de ce plan de sociologie, avant d'en montrer les avantages.

\section{Contenu de la morphologie sociale}

Cette division reste la même que dans le plan habituel. La morphologie sociale est au fond la mieux constituée de toutes les parties de la sociologie, et en elle les deux plans coïncident. Mais il suit de là qu'elle ne doit pas être entendue simplement à la façon des morphologies animale ou végétale.

Plus encore qu'un organisme dont une coupe immobilisatrice peut isoler un tissu ou dont l'anatomie résèque un organe, une société est dans le temps, dans le mouvement et dans l'esprit. Même sa structure matérielle est dans un tel perpétuel changement, ou plutôt une photographie instantanée y surprend tellement d'âges divers, deux sexes, tant de provenances, que vouloir séparer ce mouvement de cette structure, cette anatomie de cette physiologie serait rester dans l'abstraction pure. Il y a même des sociétés, nous l'avons démontré, qui ont plusieurs structures se succédant avec les saisons; d'autres sont composées d'éléments divers, dont quelques-uns eux-mêmes ont des structures diverses et variables, par exemple : ici une population maritime où les mâles sont souvent au loin; ailleurs, des groupes comme (ceux qu'on appelle pittoresquement en Amérique les «Hobo ») ces cheminots qui passent l'hiver en ville; ailleurs encore ce qu'on appelle plus techniquement la population flottante; tous ces genres de groupements et bien d'autres doivent être étudiés en eux-mêmes et dans leurs mouvements. De même l'étude de la ville ne peut être séparée de son histoire, ni de celle des origines de la population. Enfin, si les hommes se groupent en sociétés, villages et hordes, c'est parce qu'ils le veulent et des idées interviennent ici aussi. La

cependant, de dissiper toute amphibologie par l'emploi d'adjectifs, en disant : structure sociale, structure matérielle. 
morphologie sociale ne doit donc pas être comparée seulement à la morphologie des biologistes.

Disons donc, autrement, qu'elle étudie le groupe en tant que phénomène matériel ${ }^{1}$, Elle comprend et devrait rebrasser en elle-même tout ce que l'on confond ou divise arbitrairement sous le nom : de statistique (exception faite des statistiques spéciales qui relèvent de l'étude des institutions : morales, économiques, etc. ; exception faite aussi des statistiques somatiques, stature, etc. qui relèvent de l'anthropologie somatologique); sous le nom de démographie; sous le nom de géographie humaine ou anthropo-géographie ou géographie historique, ou géographie politique et économique; elle comprend aussi l'étude des mouvements de la population dans le temps et dans l'espace : natalité, mortalité, âge; alternatives, flottements des structures; mouvements et courants migratoires; elle comprend aussi l'étude des sousgroupes de la société en tant qu'ils sont ajustés au sol. C'est sur cette solide base que doit s'édifier un jour une sociologie complète. Et cette base très large, de masses et de nombres, peut être graphiquement figurée, en même temps que mathématiquement mesurée, La morphologie sociale est donc l'une des parties de la sociologie les plus compactes; elle peut donner les conclusions les plus satisfaisantes pour l'esprit.

\section{Contenu de la physiologie sociale}

Hors des hommes et des choses que la société contient, il n'y a en elle que les représentations communes et les actes communs de ces hommes - non pas tous les faits communs, comme manger et dormir, mais ceux qui sont l'effet de leur vie en société. Cette catégorie de faits est celle de la vie de la société. Elle constitue un système de fonctions et de fonctionnements. C'est donc encore de la structure, mais de la structure en mouvement. Mais surtout, puisqu'il s'agit de faits de conscience en même temps que de faits matériels, ce sont aussi des faits de vie mentale et morale. On peut donc les diviser en deux : 1 . les actes sociaux, ou pratiques sociales, ou institutions, dans le cas où les actes sont traditionnels et répétés en vertu de la tradition; 2. les idées et sentiments collectifs qui président ou correspondent à ces actes, ou sont tout au moins l'objet de croyances collectives. A cette division des faits correspond une division de la physiologie sociale en : 1. physiologie des pratiques, 2. physiologie des représentations.

On voit pourquoi, de même que le mot de morphologie, celui de physiologie doit être employé avec précaution. Il est toujours imprégné de biologie abstraite. Il ne faut pas non plus qu'il réveille la métaphore de l'organisme social. Enfin s'il exprime bien l'idée de la vie et du mouvement des hommes en sociétés, en réalité la physiologie des mœurs, des pratiques, des actes et des courants sociaux, il a le tort de ne pas exprimer clairement ce qu'il y a de conscient, de sentimental, d'idéal, de volontaire et d'arbitraire dans les poussées et dans les traditions de ces collectivités d'hommes que sont les sociétés.

Il serait facile de parler ici de psychologie collective au lieu de physiologie sociale. A un point de vue même ce serait un progrès. Car cette expression ferait bien sentir que toute cette partie de la sociologie est d'essence psychologique, que tout s'y traduit en termes de conscience, de psychologie si l'on veut dire - à condition que l'on

1 Cf. Durkheim, Année sociologique, 2, p. 530 s. 
comprît bien que celles-ci forment des communautés de conscience, qu'elles sont des consciences vivant en commun, dirigeant une action commune, formant entre elles un milieu commun. Voilà ce qu'on peut entendre par psychologie sociale. Seulement alors, si l'on y réduisait toute la physiologie sociale, toute la partie matérielle des faits de physiologie disparaîtrait de l'horizon : la transformation des idées et sentiments en actes et mouvements des individus, leur perpétuation en objets fabriqués, etc., leur fréquence elle-même. Et toute la recherche serait faussée. En effet, on laisserait échapper ainsi à la considération les deux caractéristiques par lesquelles tout fait social se distingue des faits de psychologie individuelle : 1. qu'il est statistique et nombré (nous répétons cette observation et y reviendrons encore) étant commun à des nombres déterminés d'hommes pendant des temps déterminés; et, 2. (ce qui est inclus) qu'il est historique. Car à propos de ce dernier signe, il faut bien spécifier que tout fait social est un moment d'une histoire d'un groupe d'hommes, qu'il est fin et commencement d'une ou plusieurs séries. Disons donc simplement : tout fait social, y compris les actes de conscience, est un fait de vie. Le terme de physiologie est compréhensif; il ne préjuge rien; gardons-le,

D'ailleurs, de même que nous avons essayé de purger de toute mixture biologique le terme de physiologie, de même nous essayons de préserver cette division de la physiologie sociale elle-même, entre physiologie des actes collectifs et physiologie des représentations collectives; essayons de la dégager de toute compromission psychologique. Même en psychologie, la classification correspondante est, depuis Münsterberg, l'objet de discussions passionnées. Si nous nous en servons, c'est au nom de l'usage commun. La sociologie a intérêt à n'emprunter que les mots du langage courant, mais elle doit leur donner un sens précis et à elle. Des mots de ce genre n'ont que peu d'inconvénient si l'on sait précisément ce qu'ils connotent. Or, tout dans le règne social, se place dans un autre plan, selon d'autres symétries, avec d'autres attractions que dans le règne de la conscience individuelle. Les mots : actes, représentations n'ont donc pas la même valeur; l'opposition des faits qu'ils désignent n'ont donc pas la même portée qu'en psychologie.

\section{L'intrication du mouvement et de la représentation est plus grande dans la vie} sociale.

En effet, une peine, un suicide, un temple, un outil, sont des faits matériels, comme le commerce ou la guerre. Ce sont cependant aussi des faits moraux, ou religieux, techniques, économiques, généraux. Le comportement de l'homme en tant que sociable, est donc encore plus lié à la conscience collective que le comportement individuel ne l'est à la conscience individuelle. Un acte social est toujours inspiré. Les idées peuvent y dominer même au point de nier la vie des individus, aboutir même à des destructions de peuples ou à la destruction du groupe : ainsi un siège désespéré, la résistance d'un groupe de mitrailleurs. Inversement, en tant que social, un fait est presque toujours un acte, une attitude prise. Même une négation d'acte, une paix, absence de guerre, est une chose; vivre sans procès est agréable; un tabou, un rite négatif, un commandement d'étiquette est un acte : si je ne vous dépasse pas, c'est que je me retiens de marcher. Même les représentations collectives les plus élevées n'ont d'existence, ne sont vraiment telles que dans la mesure où elles commandent des actes. La foi, quoi qu'en disent les théologiens de certaines Églises, de certaines hérésies, et certains littérateurs qui prennent les dires pour les faits, n'est rien sans les œuvres. Elle est en elle-même une œuvre, la recherche d'un état mental, d'une confiance, d'une révélation. Même chez les quiétistes parfaits elle implique une prise 
d'attitude ». le quiétisme lui-même, ce comportement négatif que l'on voudrait bien faire prendre pour une idée, mais qui consiste à vider volontairement l'âme de tout acte et peut-être de toute idée. Cette liaison intime de l'acte et de la représentation est fatale dès qu'en dehors de la pure théorie mystique, il s'agit de faits sociaux. Il y a à cela une raison le caractère collectif et par conséquent statistique des faits sociaux. Il faut qu'ils se rencontrent une ou plusieurs fois chez plusieurs individus vivant en société. Par conséquent, n'est sûrement collectif, même quand c'est une représentation pure, que ce qui se matérialise à un degré, même très lointain : par exemple dans un livre, dans le comportement d'une collectivité. Inversement, nulle part, même dans l'art et dans l'exercice le plus désœuvré de la mystique et de l'imagination ou de la science soi-disant pure, il n'y a ni idéation ni sentimentalisation (Einfühlung) dignes du nom de collectives sans qu'il y ait au moins communication, langage; sans qu'il y ait un minimum d'actes collectifs, de répétitions, d'imitations, d'autorité, et, nous ajouterons, sans une fréquence minima d'images représentées aux esprits, d'appréhensions simultanées ou identiques de certains aspects, de certaines formes (Gestalt) des choses, des idées et des actes qui font l'objet de la représentation collective. Ainsi, en sociologie comme en psychologie, nous ne sommes sûrs qu'il y a représentation que quand il y a comportement. Mais aussi, en sociologie plus sûrement qu'en psychologie, un comportement même négatif et purement inhibitoire, n'est pas un pur tropisme. Ce qui est vrai en psychologie l'est cent fois plus encore en sociologie, et encore plus vérifiable : puisque nous savons par expérience que la conduite de nos concitoyens a les mêmes raisons d'être que la nôtre, en tant qu'elle est d'importance sociale. Donc, au lieu d'opposer comme on fait communément représentation et acte, nous dirons plutôt représentation et comportement, représentation collective et comportement collectif. Et nous n'isolerons qu'exceptionnellement les uns des autres.

Il faut convenir que cette division de la physiologie sociale en physiologie des actes et physiologie des représentations ne doit pas être considérée comme une règle. Elle est simple, claire, distincte, provisoirement nécessaire pour nous. Ceci ne prouve pas qu'elle soit adéquate à toute la matière étudiée. Dans l'état actuel de la psychologie et de la sociologie, nous ne savons qu'opposer les mouvements sociaux des hommes - qui sont de la matière, du temps et de l'espace, comme les corps et les autres mouvements des corps des individus - et la conscience sociale, les états de conscience sociale qui sont dans cette société - ou plutôt les représentations collectives qu'on trouve chez les individus groupés. Ainsi le psychologue abstrait les mouvements du corps de la pensée qu'ils traduisent. Mais le fait concret, complet, c'est le tout : corps et âme. Dans la plupart des cas la question que pose un fait social, par exemple la promulgation d'une loi, ne porte ni seulement sur les concepts et les sentiments collectifs d'une part, ni seulement sur les actes et leurs sanctions d'autre part, elle porte sur le rapport des uns et des autres, et même encore plus sur des faits qui dépassent ce rapport, par exemple sur l'idéal et le normal, sur les moyennes et les réalités que l'on peut nombrer dans cette société, mais que nous savons encore fort mal appréhender. 


\section{AVANTAGES DE CETTE DIVISION}

$\underline{\text { Retour à la table des matières }}$

Sous réserve de ces observations, cette division de la sociologie ne présente aucun inconvénient. Elle est dégagée de toute métaphysique et de tout alliage d'autre science. Elle ne contredit rien, car on peut et doit l'employer concurremment avec la division en sociologie générale et sociologies spéciales, ne fût-ce que pour vérifier, recouper la recherche à tout moment. Ces deux divisions se tolèrent nécessairement l'une l'autre. Nous allons même voir comment celle-ci permet de retrouver la division en sociologies spéciales. Enfin elle ne présente que des avantages.

Le principal, répétons-le, c'est qu'elle est complète. Elle n'omet rien. Dans une collectivité il n'y a évidemment que ces trois groupes de phénomènes collectifs : la masse des individus, leurs actes et leurs idées.

Elle est claire et distincte. Elle ne divise rien qui ne soit parfaitement divisé dans la réalité.

Elle risque aussi d'être plus exacte qu'aucune autre, plus adaptée aux faits. Car elle est profondément, exclusivement concrète, calquée seulement sur des signes patents : une structure matérielle, des mouvements des groupes, des actes, cela se voit; des représentations des individus groupés cela se dit, cela se sait, même cela se voit à travers les pratiques sociales.

Ensuite il ne faut pas se laisser arrêter par les termes abstraits que nous employons - cette division, globale cette fois, est éminemment réaliste : elle présente d'un coup la réalité. Ce qu'il faut décrire, ce qui est donné à chaque instant, c'est un tout social intégrant des individus qui sont eux-mêmes des touts. Prenons pour exemple un fait moral important. Choisissons même un de ceux qui peuvent ne pas se répéter. Car il est des faits sociaux extraordinaires, non traditionnels dans la vie des sociétés : une grande émigration, une guerre, une panique sont des événements auxquels ne manquent ni le caractère historique ni le caractère statistique du fait social. Ils sont tout à la fois morphologiques, moteurs, idéaux. Descendons même jusqu'à l'analyse historique et statistique de cas particuliers englobés dans un phénomène moral, par exemple dans le suicide; considérons tel ou tel suicide, de telles gens, de tel âge, en telle et telle société : on arrive presque à rejoindre l'individu complet. Ainsi encore, un fait que nous venons d'étudier ${ }^{1}$, la suggestion collective de la mort (cette façon dans certaines populations dont les gens se laissent mourir parce qu'ils croient avoir péché ou parce qu'ils se croient enchantés) met à nu non seulement la moralité et la religiosité de ces hommes, mais le rapport de celles-ci avec la vie elle-même et le goût de la mort; c'est donc la totalité biologique que rencontre la sociologie. Ce qu'elle observe partout et toujours, c'est non pas l'homme divisé en compartiments psychologiques, ou même en compartiments sociologiques, c'est l'homme tout entier. Et c'est en suivant une pareille méthode de division des faits qu'on retrouve cet élément réel et dernier.

Enfin un pareil plan pose les problèmes en termes de sociologie pure, c'est-à-dire : en termes de nombre, d'espace et de temps, en termes de nature des idées et des

$1 \quad$ Journal de psychologie, 1926. 
actions, enfin et surtout en termes de rapports, de fonctions. Ce faisant, il rend plus claire la nature de la sociologie, plus fine et plus limité son domaine.

Car ce qui est vrai des fonctions spéciales des organes d'un vivant est encore plus vrai, et même vrai d'une tout autre vérité des fonctions et fonctionnements d'une société humaine. Tout en elle n'est que relations, même la nature matérielle des choses; un outil n'est rien s'il n'est pas manié. Revenons à notre exemple familier une industrie n'est pas seulement chose technique, il faut la considérer à toutes sortes d'autres points de vue : elle n'existe que parce qu'elle a un rendement économique, parce qu'elle correspond à un marché et à des prix; elle est localisée ici ou là pour des raisons géographiques ou purement démographiques, ou même politiques ou traditionnelles; l'administration économique de cette industrie appartient à tel ou tel pour des causes de droit; elle peut ne correspondre qu'à des arts esthétiques ou à des sports : etc. Tout, dans une société, même les choses les plus spéciales, tout est, et est avant tout, fonction et fonctionnement; rien ne se comprend si ce n'est par rapport au tout, à la collectivité tout entière et non par rapport à des parties séparées. Il n'est aucun phénomène social qui ne soit partie intégrante du tout social. Il l'est non seulement à la façon dont notre pied ou notre main ou même un viscère plus ou moins essentiel sont partie de nous-mêmes, mais - quoique cette comparaison avec les fonctions physiologiques soit encore insuffisante et quoique l'unité des phénomènes sociologiques soit encore supérieure - à la façon dont un état de conscience ou une partie de notre caractère sont non pas une partie séparable de notre moi, mais nousmême à un moment donné. Tout état social, toute activité sociale, même fugitive, doivent être rapportés à cette unité, à ce total intégré, d'un genre extraordinaire : total des corps distraits des hommes et total des consciences, séparées et cependant unies : unies à la fois par contrainte et volition, par fatalité et liberté. Car ce qui les rassemble et les fait vivre en commun, ce qui les fait penser et agir ensemble et à la fois, c'est un rythme naturel, une unanimité voulue, arbitraire même, mais, même alors et toujours, nécessaire.

Ainsi se trouve justifiée l'unité de la sociologie par une vue claire de son objet. Une note qui va suivre insistera sur cette unité à propos de livres récents. Mais dès maintenant, nous tenons à rappeler que c'est là le principe le plus fécond de la méthode de Durkheim. Il n'y a pas des sciences sociales, mais une science des sociétés. Certes on doit isoler chaque phénomène social pour l'étudier; l'explication d'un phénomène social ne peut être cherchée que dans d'autres phénomènes sociaux; mais ceux-ci ne sont pas nécessairement du même ordre, par exemple religieux, moral ou technique, que lui. Ils sont même très souvent de tout autre nature. Hors de la morphologie sociale qu'il faut distinguer et séparer pour mettre en relief sa valeur explicative, toutes les autres sections de la sociologie, les sociologies spéciales ou sciences sociales ne sont, de ce point de vue, que des parties de la physiologie sociale. Celle-ci peut être assez aisément répartie sous divers titres : religions, mœurs, économie, arts, beaux-arts et jeux, langage. Mais la sociologie est là pour empêcher d'oublier aucune des connexions. Car l'explication n'est complète que quand on a décrit, par-dessus les connexions physiologiques, les connexions matérielles et morphologiques.

Autrement dit, il ne faut jamais séparer les diverses parties de la sociologie, ni plus spécialement de la physiologie sociale, les unes des autres. Les phénomènes sociaux ont entre eux les rapports les plus hétéroclites. Coutumes et idées poussent en tous sens leurs racines. L'erreur est de négliger ces anastomoses sans nombre et profondes. Le principal but de nos études est précisément de donner le sentiment de 
ces liens les plus divers de cause et d'effet, de fins, de directions idéales et de forces matérielles (y compris le sol et les choses) qui, en s'entrecroisant, forment le tissu réel, vivant et idéal en même temps, d'une société. Voilà comment une étude concrète de sociologie, tout comme une étude historique, dépasse toujours normalement les sphères même étroitement fixées d'une spécialité. L'historien des religions, du droit et de l'économie, doit souvent sortir des limites qu'il se trace. Et cependant cet élargissement enrichit les études les plus étroitement limitées. Ainsi encore on comprendra chaque institution une à une, en la rapportant au tout; au contraire chacune isolée dans sa catégorie mène à un mystère si on la considère à part. Le moraliste trouvera toujours que nous n'avons pas «fondé » la morale; le théologien que nous n'avons pas épuisé la « réalité », «l'expérience » religieuses; l'économiste restera pantois devant les « lois » qu'il croit avoir découvertes et qui ne sont en réalité que des normes actuelles d'action. Au contraire, le problème change si on prend toutes ces parties ensemble, si on va alternativement du tout aux parties et des parties au tout. Il est permis alors, honnêtement et loyalement, de faire espérer qu'un jour, une science, même incomplète, de l'homme (une anthropologie biologique, psychologique, sociologique) fera comprendre, par toutes les conditions où l'homme a vécu, toutes les diverses formes ou au moins les plus importantes de celles qu'ont revêtues sa vie, son action, sa sentimentalité et son idéation.

Tels sont les avantages généraux de ce plan de travail. Chaque partie de ce plan possède aussi son utilité.

En particulier la division des phénomènes de la physiologie sociale a déjà cet avantage considérable : elle est rigoureusement concrète. Elle permet de poser en général tous les problèmes avec un minimum d'abstraction. Elle n'isole jamais les comportements collectifs des états de conscience collective correspondants. Et elle n'isole ni les uns, ni les autres ni du nombre, ni de la structure du groupe où on les constate.

D'abord, elle rassemble entre elles toutes les représentations et toutes les pratiques collectives, pavant ainsi la voie à une théorie générale de la représentation et à une théorie générale de l'action. En effet, les représentations collectives ont plus d'affinités, plus de connexions naturelles entre elles, bien souvent, même qu'avec les diverses formes de l'activité sociale qui leur sont une à une spécialement correspondantes. Une notion, un mot, comme l'idée et le terme de cause, sont non seulement en relation avec la religion, le droit, la technique, le langage, ils sont le total de ces relations. Même l'idée, toute la notion de cause touche la notion philosophique des valeurs, par exemple dans les jugements de valeur qui composent la magie et la religion, comme elle touche les débuts de la logique formelle en divination et en procédure. On pourrait faire d'autres observations sur la notion de faute - juridique, religieuse et, professionnelle à la fois - chez les Maoris ou même les Berbères. Les mythes - autre exemple - sont pleins de principes de droit. Et ainsi de suite. Il est dangereux de ne pas apercevoir, de ne pas rechercher systématiquement ces rapports.

De même les pratiques se tiennent souvent la main et sont moins séparées les unes des autres, que des diverses notions qui, plus ou moins consciemment, leur président. La peine est dans de nombreuses sociétés, autant une expiation ou un paiement qu'un 
acte de justice. Toute propriété est un acte économique, même celle d'un rituel. Ces observations peuvent être multipliées sans fin.

Enfin, séparant mieux les deux groupes de faits qui sont fonction l'un de l'autre, les représentations collectives et les pratiques collectives, cette division fait mieux apercevoir les rapports qui les unissent, en particulier, leurs relations indirectes et cependant intimes. Elle postule qu'il n'y a pas de représentation qui n'ait à quelque degré un retentissement sur l'action et qu'il n'y a pas d'action pure. Extérieurement le conte, celui du peuple et de la tradition, n'est que littéraire. Intérieurement, si on analyse ses mécanismes et ses thèmes, on s'aperçoit qu'il est plein de souvenirs d'anciennes pratiques, qu'il correspond à des superstitions populaires, à des règles de présage plus ou moins vivantes, etc. De même, la science apparaît à première vue comme purement idéale, la technique comme exclusivement pratique. Mais si on s'obstine à chercher les notions qui président à l'une et les mouvements que commande l'autre, on s'aperçoit vite que les deux sont dominées par une unité naturelle. La science dirige la technique qui est une science appliquée, et la technique dirige la science, car elle lui pose des questions. De même, le langage, de ce point de vue, apparaît comme chose immédiatement d'action autant que de pensée, plus même peut-être. Et le problème que les linguistes débattent se pose en termes clairs.

En dernier lieu, la morphologie sociale étant bien isolée de la physiologie, le bloc matériel de la société étant bien distingué de son épanouissement physiologique et psychologique, on peut apercevoir la solution du difficile problème des rapports entre la structure matérielle des sociétés d'une part, les actes et représentations de ces sociétés, d'autre part. Les faits que Durkheim découvrit, mais qu'il eut tant de peine à démontrer dans sa Division du travail aux philosophes qui n'y croyaient pas et aux économistes qui s'en réservaient l'étude par trop partielle, sont pour ses successeurs et seront, pour la prochaine génération de sociologues, l'évidence même. Le nombre, la densité de la population, l'intensité de la circulation et les relations, les divisions d'âge, de sexe, etc., l'état de santé, etc., apparaissent, comme ils sont, en rapport direct avec tous les phénomènes de l'activité sociale. De là, par l'intermédiaire des activités, on peut voir se dégager du groupe lui-même, dans sa structure même, les grands processus de sentiments, de passions, de désirs, les grands systèmes de symbolismes, d'images, d'idées, de préjugés, les grands choix, les grandes volitions des collectivités. Redescendant l'échelle, on peut voir, comment c'est autour d'idées, de sentiments, de traditions, de constitutions, que viennent se grouper les hommes. Et l'on peut parcourir le chemin inverse. Du spécial au général, du matériel à l'idéal, les chaînes d'analyse et de synthèse apparaissent ainsi continues.

\section{EMPLOI SIMULTANÉ DES DEUX MÉTHODES DE DIVISION}

\section{$\underline{\text { Retour à la table des matières }}$}

De cette nouvelle division superposée à l'ancienne, les divisions spéciales ne souffriront pas. Au contraire, grâce à cette étude systématique qui les assouplit, elles sortiront enrichies et éclairées, et surtout légitimées. Elles se replacent mieux, s'ordonnent, se distribuent mieux. Elles se retrouvent et ne se préjugent plus. En effet, dans cet ordre, les importantes questions de rapports de dépendance et d'indépendance des différents phénomènes sociaux passent au premier plan. Elles sont facilement 
tranchées, alors que jusqu'ici, abordées une à une par les diverses spécialités, elles sont encombrées de mots et de préjugés. Car elles offrent pour celles-ci de graves écueils.

Rien de plus simple que la définition du phénomène social et rien de plus difficile que celle des diverses catégories de phénomènes sociaux. La distinction est souvent fort utile et ne tient qu'à des différences de points de vue sur la même chose. Ainsi la théologie morale se sépare difficilement de la morale tout court; l'honnête du rituel, et inversement. Les règles d'appropriation sont-elles l'expression ou sont-elles le fondement de l'économie? on en discute. Suivant l'angle, une industrie est un phénomène économique ou un phénomène technique; elle peut être bien autre chose : la cuisine d'un bon restaurant est aussi un phénomène esthétique. Une vue de l'ensemble peut éclairer ces problèmes et faciliter ces divisions. Elle en fait aussi sentir les relativités. Car il peut y avoir et il y a sans doute, dans la société, des phénomènes importants que nous ne savons pas encore poser à leur véritable place. Nous savons à peine réserver celle que nous gardons pour eux.

Cette étude systématique des rapports permet non seulement de situer mais de « déduire » les divisions classiques de physiologie sociale. Il faut utiliser à leur propos le procédé que $\mathrm{M}$. Meillet a employé ici même ${ }^{1}$ au sujet du sens des mots : voir les groupes divers qui s'inspirent d'une même notion, font en même temps ou successivement les actes de différents sens, comme ils se servent d'un seul mot. La notion d'efficace est commune à bien des parties de la sociologie : à la technique et à la religion en particulier; on en voit cependant, même si on admet une origine commune, les divers points d'application. Les Grecs opposaient la loi à la nature, le [texte en grec] à la [texte en grec] en droit, en religion, en art, en esthétique. La notion de règle est appliquée par la science des mœurs et par la science économique. On saisit cependant la différence importante de ces deux façons de concevoir la même chose, la même attitude sociale. Une propriété est une richesse et inversement; cependant on conçoit la relation des deux termes. Peu de sujets sont plus passionnants que ceux-ci. C'est sur les confins des divisions de la sociologie, comme sur les confins de toutes les sciences et parties des sciences que s'opèrent normalement les plus grands progrès. Parce que c'est là qu'on saisit les jointures des faits et que l'on sent le mieux les oppositions de points de vue.

Naturellement il est d'autres progrès, notamment ceux auxquels Durkheim et ses collaborateurs semblent avoir le plus travaillé. Ils consistent à approfondir chacune des diverses sciences sociales que la sociologie groupe. Mais même ces progrès conduisent selon nous à dépasser les limites si vastes et pourtant encore étroites, du droit de l'économie, de la religion, etc. Ils consistent même souvent dans une simple vue des raisons historiques complexes d'un fait simple. Toute recherche profonde met à nu, sous le froid des institutions, ou sous le flottement des idées, le vivant ou le conscient tout entier, le groupe d'hommes. Dans un va-et-vient constant, en passant du tout de la société à ses parties (groupes secondaires), aux instants de sa vie, aux types d'action et de représentation; dans une étude spéciale du mouvement des parties, jointe cependant à une étude globale du mouvement du tout, doit se faire non seulement le progrès de la sociologie générale, mais celui même des sociologies spéciales. Ou plutôt, de même qu'il n'y a qu'une physique, peut-être même qu'un phénomène physique ou physico-chimique apprécié par divers sens, de même il n'y a, encore plus évidemment, qu'une sociologie, parce qu'il n'y a qu'un phénomène

1 «Comment les mots changent de sens ». Cf. Année sociologique, 9. 
sociologique : la vie sociale qui est l'objet d'une seule science, laquelle l'approche de divers points de vue. Et ces points de vue sont au fond fixés eux-mêmes par l'état historique des civilisations des sociétés, de leurs sous-groupes, dont notre science est elle-même le produit, et de l'observation desquels elle est partie. Par exemple il n'est pas sûr que si nos civilisations n'avaient déjà distingué la religion de la morale, nous eussions pu nous-mêmes les séparer. Ainsi ces divisions concrètes qui semblent opposées aux sociologies spéciales fournissent des méthodes pour les approfondir en elles-mêmes.

Il est en particulier un moyen excellent d'expliquer ces divers points de vue auxquels l'homme s'est considéré lui-même et s'est fait lui-même, et auxquels correspondent les sociologies spéciales. Celles-ci n'existent que parce que les principales activités et idéations auxquelles elles correspondent se sont divisées au cours de la très longue évolution cent et cent fois millénaire de l'humanité. Mais, si elles se sont divisées, c'est que, par rapport à elles, au moins de façon momentanée, les gens de ces sociétés se sont divisés eux-mêmes. Nous ne sommes pas toujours artisans ou toujours religieux, mais quand nous le sommes, nous le sommes généralement dans un atelier ou dans une église. Les activités sociales ont abouti, dans nombre de cas, à diviser les sociétés en de nombreux groupements variés, plus ou moins fixes. L'étude de ces groupements ou sous-groupes est, sinon la fin de la démonstration sociologique, du moins l'un des guides les plus sûrs. Pour comprendre les diverses physiologies sociales, il n'est rien de tel que de comprendre les diverses structures sociales auxquelles elles correspondent.

Il n'est pas de société connue, ou supposée connue, si basse qu'elle soit, où il n'y ait eu un minimum de répartition des individus. Ce fut une erreur de génie de Morgan d'avoir cru retrouver ce fait : la horde de consanguins; et ce n'est qu'une hypothèse de Durkheim mais, à notre sens, une hypothèse nécessaire, celle qui suppose, à l'origine de toutes nos sociétés, des sociétés amorphes. L'opposition des sexes et des générations et, très tôt, l'exogamie, ont divisé les sociétés. Mais dès qu'on entre dans l'histoire ou l'ethnographie, sans doute dès une préhistoire assez ancienne, on trouve des sociétés divisées encore, d'une autre façon : en moitiés exogames, plus exactement en deux clans exogames primaires, ou phratries, et en clans dans ces phratries, et en familles; et, d'autre part, on voit déjà poindre çà et là des noyaux de ce qui sera un jour la corporation religieuse et déjà de ce qui est la corporation magique; on voit des sortes de chefferie civile, des ateliers avec leurs techniciens, des bardes - nous ne faisons allusion ici qu'à ce que l'on constate dans les sociétés australiennes, les plus primitives de celles que nous connaissons, mais infiniment moins simples qu'on n'a l'habitude de nous les représenter. Aussi l'on peut poser la règle suivante : toute activité sociale qui, dans une société, s'est créée une structure et à laquelle un groupe d'hommes s'est spécialement adonné, correspond sûrement à une nécessité de la vie de cette société. Celle-ci ne conférerait pas la vie et l'existence à cet « être moral »ou, comme on dit en droit anglais, à cette « corporation », si ce groupe, même temporaire, ne répondait pas à ses attentes et à ses besoins.

Il n'est pas absolument forcé que ces structures soient permanentes; elles peuvent ne durer qu'un temps, et réapparaître plus tard, souvent suivant un rythme. Surtout dans les sociétés qui ont précédé les nôtres ou qui les entourent encore (j'entends toutes celles qui n'appartiennent pas à l'Asie et à l'Europe et à la branche hamitique de l'Afrique du Nord), les hommes peuvent s'organiser ainsi, sans se répartir perpétuellement en groupes fonctionnellement différents. Par exemple dans nombre de sociétés anciennes ou même contemporaines, à certains moments de la vie publique, 
les citoyens se sont répartis en classes d'âge, en confréries religieuses, en sociétés secrètes, en troupes militaires, en hiérarchies politiques. Toutes ces organisations sont différentes des phratries, clans et familles qui pourtant subsistent. Elles se confondent souvent avec ces derniers et souvent entre elles. C'est que ce sont ces groupements qui, en somme, sont chargés de telle ou telle fonction. Ou plutôt celle-ci n'est que la vie de ce groupement. Et celui-ci est soutenu, autorisé, doué d'autorité au fond, par la société tout entière. Elle abdique en lui, lui délègue sa force par rapport à tel ou tel but. Ainsi dans les sociétés nigritiennes proprement dites comme dans beaucoup de mélanésiennes, la justice est souvent l'œuvre de sociétés secrètes.

L'étude de ces groupements occasionnels, permanents ou temporaires est nécessaire, par-delà l'étude exclusive des représentations et des actes, pour les faire comprendre les uns et les autres. C'est le fonctionnement de ces groupements qui décèle quel groupe pense et agit, et comment il pense et agit; c'est ce fonctionnement qui dévoile pourquoi la société s'en remet à lui de cette pensée et de cette action, pourquoi elle se laisse suggérer par lui, pourquoi elle lui donne mandat d'agir. L'analyse se trouve terminée quand on a trouvé qui pense et qui agit et quelle impression cette pensée et cette action font sur la société dans son ensemble. Même, de ce point de vue, quand, dans des cas assez rares, c'est la société tout entière qui sent et réagit on pourrait presque dire qu'à ces moments, elles agit comme si elle formait un groupe spécial; ce qui est évident, lorsque par exception pour quelques jours ou semaines, la congrégation sociale tout entière peut être formée, par exemple, dans certaines sociétés australiennes, américaines.

Il y a donc une sorte de lieu géométrique entre les phénomènes physiologiques et les phénomènes morphologiques : c'est le groupe secondaire, la structure sociale spéciale qui reste relativement isolée. Il y a une sorte de morphologie mixte. Elle aide à déterminer ces groupes secondaires - les organes divers de la vie sociale, dont la séparation permet de séparer les diverses sociologies spéciales; celles-ci étant au fond toutes (sauf la morphologie pure) des parties de la physiologie sociale. Celle-ci comprend donc, elle aussi, l'étude de certaines structures. C'est même en décrivant celles-ci, en voyant comment l'homme se comporte à l'église, au marché, au théâtre, au prétoire, que se font au mieux les sociologies spéciales. Ce que nous proposons c'est que l'on fasse pour toutes les différentes structures sociales et leurs activités ce que l'on n'a fait jusqu'ici à fond que pour le clan et la famille. Cette étude des groupes secondaires, des milieux dont est composé le milieu total, la société, celle de leurs variations, altérations, de leurs réciproques actions et réactions est, à notre avis, une des choses non seulement les plus souhaitables mais les plus faciles et les plus urgentes qui soient. C'est là, encore plus que dans la pratique sociale - l'institution étant toujours à quelque degré figée - que se constate la véritable vie, matérielle et morale en même temps, le comportement du groupe. Même les processus collectifs d'idéation, de représentations, peuvent être traités de cette façon. Elle semblera bien terre à terre et même bien lointaine et inadéquate à quiconque est amoureux de vague et d'idéal. Ce sont, à notre avis, les chercheurs d'ineffable qui se trompent. Au contraire de ce qu'ils disent, on est sûr qu'il y a mythe ou légende, pensée forte et ancrée, quand il y a pèlerinage de saints, fête, clans ou confréries attachés à ces lieux saints. Le jeu des idées collectives est sérieux quand il se reflète dans les lieux et dans les objets, parce qu'il se passe dans les groupes que d'ailleurs ce jeu crée, dissout et recrée sans cesse.

Ainsi cette division des phénomènes sociaux en morphologiques et physiologiques et celle des phénomènes physiologiques en représentations et en actes 
collectifs peut s'appliquer utilement à l'intérieur des différentes sociologies spéciales. Peut-être même faut-il s'en servir obligatoirement quand on étudie séparément les phénomènes sociaux cloisonnés en religieux, juridiques, économiques, etc. Les spécialités découpent les grandes classes de faits pour ainsi dire en piles verticalement disposées; au contraire on peut aussi diviser ces sections en tranches pour ainsi dire horizontales, par degrés, par couches d'idéation croissante ou décroissante, de matérialisation plus ou moins grande selon qu'on s'éloigne ou se rapproche de la représentation pure ou de la structure matérielle proprement dite. A notre avis, cette division fournit un principe de méthode pour l'étude de chaque grand groupe de faits. Elle constitue une sorte de preuve arithmétique que l'on a été complet. Car, à notre sens, un phénomène social est expliqué quand on a trouvé à quel groupe il correspond, et à quel fait de pensée et d'acte il correspond, qu'il soit physiologique ou morphologique, peu importe.

L'application de ce principe va de soi quand il s'agit de physiologie pure. Cependant peu de sociologues l'emploient de façon constante. Il est pourtant presque infaillible à l'usage. Il force à voir, à chercher les actes sous les représentations et les représentations sous les actes et, sous les uns et les autres, les groupes. Des séries d'institutions qui apparaissent, à la surface, comme composées exclusivement de pratiques traditionnelles ou d'actes de fabrication, comme la coutume et les techniques, sont pleines de notions que la science du droit et la technologie doivent dégager. D'autres séries de faits sociaux qui apparaissent comme purement rationnelles, idéales, spéculatives, imaginatives, ou sentimentales et ineffables, telles la musique ou la poésie et la science, sont pleines d'actes, d'activités, d'actions, d'impressions sur les sens, sur la respiration, sur les muscles ou de pratiques et de techniques.

Inversement la morphologie sociale qui sert de contrôle à la physiologie, doit être soumise à ces analyses, elle aussi. Le groupe n'apparaîtra plus jamais inerte ou inconscient. Son unité, la volonté, l'habitude de vivre en commun l'expliquent. Cellesci, en plus du rassemblement de la masse, sont faites de toutes ces multitudes d'impondérables, de tendances, d'instinct, d'imitations, d'idées communiquées, de sentiments passagers, sans parler des hérédités communes.

Ici se justifie l'idée profonde de la métaphysique, de la philosophie et même de la pensée allemandes, même du vulgaire en Allemagne : qu'une "Weltanschauung », qu'une « conception du monde » commande l'action et même l'amour. Il est juste de dire qu'autant qu'un sol et une masse, c'est une tonalité de vie qui forme toute société. La société inspire en effet une attitude mentale et même physique à ses membres et cette attitude fait partie de leur nature. Et ces attitudes de ces masses peuvent être nombrées : premier point de l'éthologie collective.

D'ailleurs, nous l'avons vu déjà, la morphologie sociale figure la société non seulement dans l'espace et le nombre, mais encore dans le temps. Elle étudie aussi des mouvements, des altérations et des dynamismes. De plus, tout comme la psychologie sociale, ou plutôt la physiologie sociale, se traduisent dans la matière humaine et, à l'occasion, dans l'espace et le temps sociaux où tout se passe, de même la structure matérielle du groupe n'est jamais chose indifférente à la conscience du groupe. Souvent les faits de morphologie sont vitaux pour elle. Par exemple, voici les frontières : on les dirait entièrement morphologiques, géographiques; mais ne sont-elles pas en même temps un phénomène moral et militaire, et pour certains peuples, pour les anciens surtout, un phénomène religieux? 
L'intérêt principal de ces observations est qu'elles permettent de faire comprendre, de systématiser et d'exiger l'emploi des méthodes quantitatives. Qui dit structures matérielles et sociales et mouvements des structures dit choses qui peuvent être mesurées. Ce lien du morphologique et du physiologique permet donc de mesurer la place considérable que devrait occuper ici, dans toutes les études de physiologie sociale, la recherche statistique. Les sous-groupes et leurs actions peuvent en effet être dénombrés. On recense les professions. Même les crimes correspondent, pour ainsi dire, au sous-groupe des criminels.

Hélas! même dans l'Année sociologique, nous sommes loin de compte. La statistique, mathématique sociale, pourtant d'origine sociologique elle-même, semble se réduire pour nous aux problèmes usuels : de la population (morphologie), de la criminologie et de l'état civil (statistique morale) et de l'économie, cette partie de nos sciences qui se vante d'être le domaine du nombre et des lois du nombre et qui l'est en effet en partie. Cette restriction de l'emploi de la statistique est inexacte. Au fond, tout problème social est un problème statistique. La fréquence du fait, le nombre des individus participants, la répétition au long du temps, l'importance absolue et relative des actes et de leurs effets par rapport au reste de la vie, etc., tout est mesurable et devrait être compté. L'assistance au théâtre ou au jeu, le nombre des éditions d'un livre instruisent sur le prix attaché à une oeuvre ou à un sport beaucoup mieux que des pages et des pages de moralistes ou de critiques. La force d'une Église se mesure au nombre et à la richesse de ses temples, au nombre de ses croyants et à la grandeur de leurs sacrifices, et, s'il faut aussi toujours considérer les impondérables en elle, ne considérer que la foi et la théologie est une non moins grave erreur que de les oublier. Manié avec prudence et intelligence le procédé statistique est non seulement le moyen de mesurer mais le moyen d'analyser tout fait social, parce qu'il force à apercevoir le groupe agissant. Il est vrai que, bien des travaux statistiques actuels eux-mêmes sont plutôt inspirés par les besoins administratifs ou politiques des États, ou bien sont mal dotés, ou mal dirigés par une curiosité mal éclairée de professionnels; ils présentent un fatras. Les vrais travaux sont encore à entreprendre, Cependant on sait déjà combien l'historien et le sociologue des générations qui viennent seront mieux armés que nous ne fûmes. Dès nos jours, dans des travaux immenses, comme ceux du « census » américain ou du « census » des Indes, l'on voit apparaître, à travers les statistiques compilées, les choses sociales en ébullition : le «chaudron de la sorcière » où se fabrique une société. Dans des études ainsi entreprises, le cadre de toutes les divisions spéciales elles-mêmes s'enrichit.

\section{UTILITÉ DE CETTE DIVISION POUR UNE SOCIOLOGIE GÉNÉRALE CONCRÈTE}

$\underline{\text { Retour à la table des matières }}$

C'est surtout au point de vue de la sociologie générale que cette division a des avantages. Elle la prépare directement. Dans cette sociologie concrète, on a donc de mieux en mieux décrit les rapports qui existent entre les divers ordres de faits sociaux considérés tous ensemble et considérés chacun séparément : morphologiques et physiologiques d'une part et, en même temps, religieux, économiques, juridiques, linguistiques, etc. C'est alors qu'on peut entreprendre de constituer vraiment une sociologie en même temps générale et cependant concrète. 
Le procédé est simple, c'est d'étudier tous ces rapports. Par un côté même, la sociologie générale consiste dans la découverte de ces rapports.

D'ailleurs ce nom de sociologie générale prête à l'erreur. Elle n'est pas le pur domaine des pures généralités, surtout des généralités hâtives. Elle est, avant tout, l'étude des phénomènes généraux. On appelle généraux ceux des phénomènes sociaux qui s'étendent à toute la vie sociale. Mais ils peuvent être tout à fait particuliers, précis; ils peuvent manquer ici et là, et être même restreints à des sociétés déterminées. Ces phénomènes généraux sont ceux : de la tradition, de l'éducation, de l'autorité, de l'imitation, des relations sociales en général, entre classes, de l'État, de la guerre, de la mentalité collective, de la Raison, etc. Nous négligeons ces grands faits et les négligerons probablement encore longtemps. Mais d'autres ne les oublient pas. Sur l'autorité, on peut citer le livre de M. Laski. Durkheim et les partisans de la Social Pedagogics traitent de l'éducation. D'autres auteurs réduisent même la sociologie tout entière à ces considérations des faits généraux : c'est le cas de Simmel et de ses élèves, celui de M. von Wiese et de sa «Beziehungslehre ». Nous ne sommes pas trop d'accord avec eux; mais ils ont raison de ne pas considérer l'étude des édifices sociaux comme relevant de la seule sociologie juridique. Sur l'État et les nécessités de son étude, nous allons revenir incessamment à propos de la sociologie appliquée et de la politique.

Une autre partie de la sociologie générale concerne les rapports que les faits sociaux ont avec les faits voisins. Or, du dernier point de vue, les rapports de la sociologie et des deux sciences immédiatement annexes, la biologie et la psychologie, deviennent visibles. Les connexions de la morphologie sociale, science du matériel humain, et de la biologie sont claires. Celles de la physiologie sociale avec la biologie le sont moins. Mais si l'on saisit que les phénomènes morphologiques sont le moyenterme-cause, les raisons d'être, qui relient les idées et les actions sociales aux faits biologiques et inversement et ceux-ci aux idées, etc., tout s'éclaire. Une population a un idéal de beauté et se crée un type physique, par l'action de cet idéal sur le mariage, sur la natalité. Une population a un nombre déterminé de fous; ces fous se suicident ou commettent des crimes suivant les saisons, suivant les quantités d'heures de jour, c'est-à-dire suivant l'action de la nature sur la longueur et l'intensité de la vie sociale. Nous ne citons que des faits bien connus.

On éclaire encore ainsi le rapport entre la psychologie et la sociologie. La psychologie des représentations, celle des actes et celle des caractères viennent se rapprocher non plus de tous les phénomènes sociologiques, mais de ceux des phénomènes sociaux correspondants : représentations et actes collectifs, caractérologie, etc. Et les problèmes de confins, si importants, où la psychologie et l'individuel jouxtent la sociologie et le social, se posent en termes de faits : ainsi ceux du langage, ceux des sentiments religieux, moraux, etc.

Citons enfin trois des parties de la sociologie générale qui, à notre avis, peuvent tout de suite bénéficier d'une méthode de ce genre. Ce sont : la théorie du symbolisme, celle de la raison et enfin celle des caractères collectifs. Les deux premières sont maintenant posées très généralement. La dernière était fort en vogue au temps de Taine. Elle est désuète maintenant, à tort à notre avis. 
Le problème de la pensée, à la fois pratique et théorique, celui de son rapport avec le langage, le symbole et le mythe, celui du rapport de la science et de la technique trouvent ici leur place normale, exacte, parce qu'on peut les considérer tous ensemble.

1. Le problème du langage et du symbole (et le problème, plus général et plus crucial, de l'expression) sort tout de suite de la spécialité où le cantonnent les linguistes et les esthéticiens et des généralités où se meuvent les philosophes et quelques psychologues. Ces deux choses essentielles, si intimement liées, connaissances et symboles de tous ordres apparaissent enfin comme elles sont : liées à la totalité des activités du groupe et à la structure même de celui-ci et non pas simplement à telle ou telle catégorie de ces activités. Car il est des symboles et des connaissances en économie comme en religion, comme en droit et non pas simplement comme en mythologie ou en art. Et le totem ou le drapeau symbolisent le groupe.

2. Plus généralement, les études de «mentalité », de «fabrication de l'esprit humain », de « construction et d'édification » de la raison, sont revenues à la mode. C'est elles que Comte avait en vue. Durkheim, Hubert, nous, d'autres et, parmi eux, M. Lévy-Bruhl, M. J. H. Robinson, les ont remises en honneur en termes précis, croyons-nous. Elles peuvent être et doivent être élargies. Au fond, elles supposent la connaissance simultanée de nombreux éléments, dans de nombreuses civilisations. Les données qui doivent entrer en ligne de compte sont esthétiques, techniques, linguistiques et non pas seulement religieuses ou scientifiques. Là aussi ce sont des mélanges qu'il faut déceler et des dosages qu'il faut faire. Et après les avoir fait, il faut rebrasser tout cela, synthétiser en termes encore plus précis. On fera ainsi apparaître le « total » dans l'histoire : l'empirique, l'illogique et le logique du début, le raisonnable et le positif du futur. Tant que les nombres ont eu une valeur mystique et linguistique en plus de leur usage technique et intellectuel; tant que les maladies ont été quelque chose de moral ou de religieux, des sanctions du péché par exemple, l'arithmétique ou la médecine avaient une autre tournure que celle qu'elles ont prise. Cependant elles existaient. Les premières pages d'Hippocrate marquent merveilleusement la révolution interne qui fit passer, un jour, en Ionie, la médecine à la science. Notre arithmétique elle-même s'est encore développée dans la recherche des carrés magiques et celle des racines mystiques, bien après Pythagore, jusqu'au XVIIe siècle. Notre pharmacopée du XVIIe, du XVIIIe siècle encore, venaient de civilisations qui mêlaient toutes sortes d'observations insolites à leur pathologie, à leur thérapeutique, mais qui avaient de fort sérieuses connaissances en pharmacie : en Arabie, en Inde, en Chine. Chez les pharmaciens comme chez les alchimistes, il y avait plus que de la foi et de l'empirisme, il y avait de la science. La raison et l'expérience intelligente sont aussi vieilles que les sociétés et peut-être plus durables que la pensée mystique. Ainsi encore en voyant l'ensemble, on prépare l'analyse de la conscience collective ;

3. Les études de mentalité ne sont au fond qu'une partie des études de civilisation et d'éthologie que nous distinguons fort mal. Il faudra un jour les séparer. Pour le moment, l'éthologie collective, si difficile à constituer, nous l'avons vu, peut les considérer d'un coup. Ces analyses de l'âme d'une société ou d'une civilisation peuvent être comparées à des analyses chimiques. Selon les vues profondes de 
Durkheim, de même que les caractères individuels, les caractères des sociétés et des civilisations sont simplement des composés d'éléments mesurables.

Des types de vie sociale plus ou moins répandus, voilà ce qu'on appelle des civilisations. Dans telle ou telle société, les principales caractéristiques de la vie sociale sont plus ou moins autochtones, proviennent en plus ou moins grande partie de sociétés plus ou moins voisines. Les sociétés sont plus ou moins fermées. Par exemple, le Moyen Age chrétien était beaucoup plus un univers, une " universitas », une catholicité que notre Europe et, cependant, les groupes qui le formaient étaient infiniment plus divers et plus nombreux. Mais ils étaient moins organiques et c'est pourquoi ils étaient infiniment plus perméables les uns aux autres, plus faibles vis-àvis des influences d'en haut; ils étaient encore sous l'impression de l'Empire romain : par suite les couches supérieures de ces nations encore mal définies, l'Église, l'Université les principales corporations, les grandes confréries, dont la chevalerie, étaient beaucoup plus internationalisées qu'aujourd'hui. Voilà pour la notion de civilisation.

Quant au caractère des gens d'une société, il singularise celle-ci. Certaines sociétés sont plus adonnées à la recherche idéale et esthétique, ou au commerce. D'autres sont plus adonnées aux arts pratiques, à l'administration et au commandement; là est l'opposition classique de Rome et de la Grèce. Notre regretté Huvelin revenait brillamment sur ce sujet à propos du droit romain. Le Dr Jung et M. Seligmann vont jusqu'à parler de psychanalyse des races et des sociétés, et même d' "introversion » et d' « extraversion » à leur propos. Ils poussent un peu loin le freudisme ou le jungisme. Ces classifications n'ont déjà pas trop grande valeur en psychologie et physiologie individuelles; elles n'en ont plus guère en sociologie. Mais elles donnent le sens de ce qu'est une science des caractères sociaux et, s'il en est, des caractères des races.

On peut en effet classer les sociétés à de multiples points de vue. Ainsi les unes sont prédominées par des éléments jeunes, par exemple la Russie, d'autres par des masses âgées, par exemple la France. D'un autre point de vue, elles sont rurales (russes) ou urbaines en majorité (anglaises), agricoles ou industrielles, etc. C'est ici qu'on pourra reprendre, en d'autres termes, mais en poursuivant les mêmes buts que M. Steinmetz ${ }^{1}$ la vaste question de la classification des sociétés ou plutôt du catalogue de celles-ci. Car c'était au fond à des dosages que M. Steinmetz s'attachait, de façon remarquable pour l'époque.

Ces classifications poussées dans le détail arriveront peut-être un jour à rendre compte de la spécificité de chaque société connue, à expliquer son type spécial, son aspect individuel. Au fond, c'est ce que font inconsciemment, mais non sans méthode les historiens, les pratiquants de l' « histoire » devenue enfin « sociale ». On arrivera peut-être même à rendre compte des idiosyncrasies et ensuite à diagnostiquer à part l'état précis, à chaque instant, de chaque société. Tous ces problèmes échapperont aux généralités et à la littérature politique ou même historique. Ainsi de même que la psychologie doit être couronnée par une « caractérologie », de même une « caractérologie des sociétés », une « éthologie collective » concrète achèvera la sociologie générale et aidera à comprendre la conduite actuelle de chaque société.

\footnotetext{
Cf. Année sociologique, 2.
} 
On saura alors, ce que nous ne savons pas, sans danger pour les sociétés ellesmêmes, poser le problème de la sociologie appliquée ou politique. On sera prêt pour sauter ce dangereux pas : le vide qui s'étend de la science sociale pure à la direction de l'action.

Mais, on le remarque, ces deux plans d'une sociologie pure ne comprennent rien qui concerne la politique. Or, à ce point, nous rencontrons des traditions contraires de sociologues respectables. Il faut nous expliquer sur cette autre discipline, la politique, que nous ne pratiquons pas.

\section{PLACE DE LA SOCIOLOGIE APPLIQUÉE OU POLITIQUE}

$\underline{\text { Retour à la table des matières }}$

L'un des principaux avantages d'une connaissance complète et concrète des sociétés et des types de sociétés, de chaque société à part, des nôtres en particulier, c'est qu'elle permet d'entrevoir enfin ce que peut être une sociologie appliquée ou politique. On doit impitoyablement éliminer celle-ci de la sociologie pure. Et cependant, seule chose qu'on puisse faire ici, on entrevoit, tout à fait séparément, quelques principes de l'application de nos sciences.

La politique n'est pas une partie de la sociologie. Les deux genres de recherches sont trop mêlés encore aujourd'hui. Nous insistons sur leur séparation. Elle est contraire à la tradition américaine, nettement « mélioriste » depuis Ward. Les sociologues américains ont généralement le sentiment aigu que les civics, les politics, le social service, le social work, en général les social forces et les ethics sont aussi leur et constituent leur domaine. Ils les confondent avec la sociologie. Au contraire nous, ici, en France, et dans l'Année sociologique, nous ne nous occupons intentionnellement pas de la politique. Nous avons pour cela une raison de principe que Durkheim a souvent indiquée et précisée : ceux qui font cette confusion entre la science et l'art se trompent et au point de vue de la science et au point de vue de l'art. Chercher des applications ne doit être ni l'objet d'une science, ni le but d'une science : ce serait fausser celle-ci, Et l'art n'a pas à attendre la science : celle-ci n'a pas pareil primat.

Mais si la sociologie doit rester pure, elle doit se préoccuper de son application. Durkheim disait qu'elle ne vaudrait pas « une, heure de peine » si elle n'avait pas d'utilité pratique. Comme toute spéculation, elle doit en effet correspondre à une technique. D'ailleurs Durkheim savait que la politique positive et la sociologie ont la même origine et sont nées du grand mouvement qui a rationalisé l'action sociale au début du XIXe siècle. En pensant à l'application de la sociologie, nous restons donc fidèles à la tradition. Le seul reproche que l'on peut faire à Comte, aux premiers élèves de Comte et à Spencer, la raison pour laquelle ils se trompaient, c'est qu'ils crurent pouvoir légiférer au nom de réflexions fort générales, de recherches fort sommaires dont ils ne savaient contrôler ni les unes ni les autres. Les économistes classiques ont échappé aux généralités, mais non à ces prétentions normatives. Il est vrai qu'ils sont plus avancés que la plupart des autres zélateurs des sciences politiques. Mais ils ne sont guère plus fondés à diriger la pratique; celle-ci, sauf sur 
certains points de législation financière et de pratique bancaire, se rit bien de leurs prévisions. - Il faut donc appliquer la science. Mais il ne faut pas confondre ses applications avec la science elle-même. Les raisons de la confusion courante sont instructives. Répétons ce que Durkheim a dit à ce sujet, en termes légèrement différents.

Si cette erreur de tant de savants est normale, c'est que la Sociologie est plus près qu'aucune autre science de l'art pratique correspondant, de la politique, du moins de celle des temps modernes. L'une et l'autre supposent que, hors de tous préjugés religieux, moraux ou autres, la société prend conscience d'elle-même, de son devenir d'une part, de son milieu d'autre part, pour régler son action. Tandis que toutes les autres pratiques et industries ont un objet matériel extérieur et extraconscient qui leur impose des attitudes auxquelles on sait d'avance que le succès peut ne répondre qu'en partie; tandis que même la pédagogie et la psychiatrie ont un autre objet que la psychologie, surtout introspective - les hommes qu'il s'agit d'observer, puis de guérir ou d'éduquer; au contraire la politique et la sociologie n'ont qu'un seul et même objet : les sociétés. De celles-ci les hommes s'imaginent tout connaître, parce qu'elles leur apparaissent comme n'étant composées que d'eux, de leurs volontés, de leurs idées malléables à volonté. Ils croient leur art souverain et leurs connaissances parfaites.

Mais c'est précisément parce que l'art, la pratique politique rationnelle et positive est si proche de la science des sociétés, que la distinction entre les deux est plus nécessaire que partout ailleurs. Il ne suffit pas de maquiller l'action à l'aide de statistiques dressées elle-mêmes sur des plans préconçus ou triturés suivant les idées des Partis et du moment, pour donner à cette action une allure non partisane, sereine, sociale, pure de tout alliage et de tout intérêt. Il ne suffit pas non plus d'être sociologue, même compétent, pour dicter des lois. La pratique, elle aussi, a ses privilèges. Même, souvent, la carence de la science est telle qu'il vaut mieux se confier à la nature, aux choix aveugles et inconscients de la collectivité. Il est maintes fois bien plus rationnel de dire qu' " on ne sait pas », et de laisser se balancer les impondérables naturels - ces choses de conscience dont on ne saisit pas à quoi de précis elles correspondent : les intérêts, les préjugés. Ceux-ci se heurtent dans les tribunaux, la presse, les bourses et les parlements; ils s'expriment dans l'éthos et le pathos des orateurs, dans les adages du droit, les proclamations des maîtres de l'heure, les ordres souverains du capital et de la religion, les mouvements de la presse, les élections plus ou moins claires. Et il vaut mieux laisser ces forces agir. L'ignorance consciente est meilleure que l'inconscience. L'aveu d'impuissance ne déshonore ni le médecin, ni l'homme d'État, ni le physiologue, pas plus que le sociologue. Ce «complexus »si riche de consciences, de corps, de temps, de choses, de forces anciennes et de forces latentes, de chances et de risques qu'est une société, devrait être traité le plus souvent comme une immense inconnue par les gens qui prétendent le diriger - alors qu'ils sont dirigés par lui, ou qu'ils tentent tout au plus d'exprimer son mouvement par les symboles que leur fournissent le langage, le droit, la morale courante, les comptes en banque et les monnaies, etc.

Ceci est dit, non pour diminuer, mais, au contraire, pour exalter l'art politique et son originalité. Le tour d'esprit du politicien, son habileté à manier les formules, à « trouver les rythmes » et les harmonies nécessaires, les unanimités et à sentir les avis contraires sont du même genre que le tour de main de l'artisan : son talent est aussi précieux, aussi natif ou aussi traditionnel, aussi empirique mais aussi efficace. La science n'est créatrice que rarement. L'homme de loi, le banquier, l'industriel, le religieux sont en droit d'agir en vertu de leurs connaissances pratiques et de leurs 
talents. Il suffit d'avoir administré ou commandé pour savoir qu'il y faut une tradition pratique, et qu'il y faut aussi une chose qu'un psychologue mystique traduirait en termes d'ineffable : un don. Aucune raison ni théorique, ni pratique ne justifie donc un despotisme de la science. Seulement cette distinction de l'art et de la science, et cette constatation de la primauté actuelle de l'art politique étant bien posées, la sociologie peut intervenir et justifier ainsi son existence... matérielle, c'est-à-dire la fonction sociale des sociologues.

\section{La « sociologie de la politique», partie de la « sociologie générale»}

D'abord, il est possible de faire la science de cet art. Et cette science des notions politiques nous regarde. Non pas ce qu'on appelle, dans certaines régions, les sciences morales et politiques : la science financière, la science diplomatique, etc. Le plus souvent, ces soi-disant sciences ne sont que de vulgaires mnémotechnies, des recueils des circulaires et des lois, moins bien digérées que les vieux codes. Elles ne sont que des catalogues de préceptes et d'actions, des manuels de formules, des recueils de maximes de la technique sociale. Indispensables certes, elles encombrent le pavé de leurs prétentions et les Écoles de leurs chaires; elles ne sont que des enseignements de pur apprentissage. Cependant, quelquefois, çà et là, on peut faire profit de leurs travaux. Des esprits puissants ont haussé leur spéculation à dégager les principes de ces arts, à démêler le genre d'activité sociale, d'esprit social, qui président au fonctionnement même de l'usage et du droit. En ce moment même, en France, des juristes, M. Hauriou, et M. Duguit, font un effort considérable pour dégager les principes du droit public. En Allemagne, les juristes moralistes, MM. Wilbrandt, Radbruch, d'autres ont à un tel point agi sur leurs pays qu'ils y ont conquis une position politique. Un certain nombre des meilleurs théoriciens de la politique en Amérique, M. Merriam en tête, sont arrivés à la sociologie, d'eux-mêmes, en partant de la pratique elle-même. Nous rendrons compte de l'œuvre de ce dernier. Ailleurs la prise de conscience a été le fait de la civilisation, de la société elle-même. Le prestige du droit romain, celui de la politique et de la morale grecques, celui de la « sagesse hindoue », celui de l'idéalisme juif, viennent de la clarté de l'esprit de ces peuples : ils démêlaient avec force, netteté, leurs visions, le symbole central des autres symboles de leur action. Les Anglais ont eu, eux aussi, leurs « prudents», ceux de la «Common Law », comme ceux de la politique, du droit constitutionnel. De Hobbes à Austin une longue série d'auteurs doit être rangée parmi les vrais fondateurs de la politique et de la sociologie. Un homme de loi anglais sait pour ainsi dire naturellement ce que c'est que le souverain. Il ne faut pas sous-estimer le bénéfice de pareils éclats du génie humain. La trouvaille et la recherche de ces prises de conscience collective, forment le meilleur fondement des études de sociologie et de politique actuelles. L'École historique et de pure observation domine enfin avec raison : or, elle puise dans les théories implicites, comme dans les théories explicites de tous les temps, le principe et la substance de ses idées.

Seulement, elle est encore trop attardée; elle ne considère que les formes et les constitutions. C'est ici que la sociologie peut lui donner une importante impulsion. Normalement, même en régime parlementaire ou réglementaire, même dans nos arts politiques qui prétendent être positifs, expérimentaux, qui essaient de se fonder sur des statistiques et des chiffres, même dans nos affaires, où l'art comptable rend tant et de si bons services, c'est cependant l'inconscient, le besoin évoquant sa satisfaction, 
c'est l'action qui dominent. Cette dernière est éclairée, certes, ni aveugle, ni mystique, pourtant elle reste inanalysable ou peu analysée. Or, il est possible de faire une théorie de l'art politique : d'abord avec l'aide de ces prises de conscience de la collectivité elle-même qui sait choisir ses dirigeants et les inspirer; puis, avec tous les procédés de l'histoire comparée, permettant l'analyse des faits; en un mot, à l'aide d'une « pragmatique » comme disait Aristote. On peut constituer une science de l'art social. Cette science commence à se constituer : elle consiste simplement à apercevoir, grâce à ces données, connues déjà en partie, comment, par quels procédés politiques, les hommes agissent, ont su ou cru agir les uns sur les autres, se répartir en milieux et groupes divers, réagir sur d'autres sociétés ou sur le milieu physique. On voit comment cette théorie de cet art fait partie d'une sociologie à la fois générale et concrète.

Cette science de l'art social, nous la plaçons dans l'Année, parmi les disciplines ressortissant à la sociologie morale et juridique, ou dans la sociologie générale. Nous avons déjà avoué ces flottements. Dans le premier cas, nous opérons ainsi sous prétexte que le phénomène de l'État est un phénomène juridique. Il est vrai : l'État, organisme politique de la société, la constitution, l'établissement d'un pouvoir souverain sont des faits juridiques et moraux. Mais ils sont sûrement davantage. Ils concourent au tout de la société et tout y concourt vers eux. Dans quelle mesure?

Nous ne savons pas le préciser, nous ne savons que le faire sentir. Les frontières de l'État, par exemple, ce point hypersensible de la société et de ]'État politique, sont de l'ordre morphologique, nous l'avons déjà dit; et ainsi de suite... L'art politique et la science de cet art doivent donc, comme la sociologie elle-même, tenir compte de tous les faits sociaux. En particulier dans nos sociétés modernes, les phénomènes économiques et morphologiques (démographiques) entrent sous sa juridiction. Tout spécialement, des choses importantes qui échappent à nos rubriques : la tradition, l'enseignement, l'éducation, en sont parties essentielles. Il faut donc rompre le cadre étroit de la théorie juridique de l'État. Il faut étendre la théorie politique à celle de l'action globale de l'État. Il faut aller plus loin, voir les sous-groupes : non seulement analyser l'action du centre, mais aussi celle de tous les groupes secondaires, volontaires ou involontaires, permanents ou temporaires, dont est composée une société. Normalement, cette théorie de l'art social élargit la politique. De ce côté, son action est un bienfait. Car si la confusion du problème de l'État, de la souveraineté, avec un problème juridique fut fatale, elle constitue une erreur de fait et une erreur pratique. Procéder de la façon habituelle mène aux pires dangers. Les fondateurs de la science positive des sociétés, fondateurs aussi de la politique positive, Saint-Simon, Comte, firent immédiatement prêter attention à cette faute. Ils avaient une certaine haine du législateur, de l'homme de loi, de l'administration, et un certain fétichisme de l' « industriel », du « savant », du « producteur ». Cette attitude est devenue traditionnelle dans le socialisme et jusqu'au bolchevisme. Évitons leur excès, car l'art de gérer et de commander et de manœuvrer légalement sera toujours essentiel à la vie en commun, même à la vie technique. Il reste que, un peu par la force des choses et beaucoup par force d'inertie, nos parlementarismes occidentaux remettent à trop de robins et de publicistes, le soin d'intérêts qui dépassent les limites de la légalité et de la bureaucratie. Il faudra donc, de toute nécessité, rompre avec la tradition antique qui a mené la politique, depuis les chancelleries lagides et romaines, jusqu'au Conseil privé des rois. Les sociétés modernes savent que bien des choses éminemment sociales ne doivent pas être remises à des fonctionnaires, à des conseillers, à des légistes. Celles qui mettent en jeu et même en question la société elle-même, comme la guerre et la paix, doivent être décidées autrement qu'autrefois. Le service principal 
que les sociologues ont rendu jusqu'à maintenant et rendront de plus en plus à la politique, par une théorie de la politique elle-même, consiste donc à faire sentir à quel degré les problèmes politiques sont des problèmes sociaux. Ils auraient par suite le plus grave tort si, pour ne pas verser dans l'erreur commune, ils restaient tous dans leur tour, s'ils s'abstenaient tous de prendre parti, s'ils laissaient la politique aux théoriciens politiciens et aux théoriciens bureaucrates. L'art de la vie sociale les concerne en particulier et transmettre une tradition, éduquer les jeunes générations, les intégrer dans une société déterminée, les «élever » et surtout les faire progresser, tout cela dépasse les limites du droit et de tout ce qu'on convient d'appeler l'Etat. La science de cet art fait donc partie de la sociologie générale, ou, dans une sociologie divisée de façon concrète, d'une partie toute spéciale de la sociologie de l'action.

\section{Sociologie et politique}

Ainsi conçue cette théorie de l'art politique est une partie essentielle de la sociologie et plus spécialement, dans nos divisions proposées, de la sociologie générale, et dans celle-ci, de la théorie des ajustements généraux. Mais cette science de l'art social, politique reste théorique. Comme le reste de la sociologie, elle a surtout pour méthode la comparaison historique ou l'analyse statistique, bien que les faits comparés soient des faits modernes. A ce titre, elle est certainement intéressante, instructive, informatrice. Mais elle n'est qu'une petite contribution à la direction réelle des sociétés actuelles. L'art de diriger une société, l'action, l'administration, le commandement sont choses autrement vitales et puissantes que cette influence indirecte de la science des sociétés. Cette action à distance est relativement peu de chose par rapport à la politique tout court. Comment pouvons-nous contribuer efficacement à celle-ci? Voilà le problème final de la sociologie.

Déjà, au contact de celle-ci, l'action politique est singulièrement agrandie : on l'entend au sens large sous son inspiration; on comprend en elle, non seulement la direction des organes de la souveraineté, mais encore le contrôle des forces financières, des industries, de l'éducation, des relations matérielles, morales et intellectuelles avec les autres nations. De plus, éclairée, rehaussée, affinée par la sociologie, cette action peut être infiniment meilleure que si on la laisse aveugle. Donc l'art politique ne doit pas être indépendant de la sociologie, et celle-ci ne doit pas se désintéresser de lui. Mais quels doivent être leurs rapports? Quelle place faut-il leur donner dans une sociologie complète? Voici quelques indications.

D'abord il faut répéter le vœu de Spencer repris par Durkheim que la connaissance de la sociologie devrait être requise pour qualifier l'administrateur et le légiste. En fait, dans de nombreux pays, la sociologie fait partie des programmes d'examens du futur fonctionnaire et de nombreuses écoles de hautes études commerciales ou administratives. De plus, en fait encore, la sociologie agit déjà clairement de nos jours sur la politique. Celle-ci a pris une attitude positive, expérimentale qui provient, plus qu'on ne croit, de nos études.

Seulement que doit être le rapport inverse? Si nous voyons clairement ce que nous devons exiger du politicien, et même du citoyen qui se doit de s'éclairer, qu'est-ce que celui-ci a à réclamer de nous? D'abord notre attention. C'est-à-dire : le public ne nous permet pas de nous occuper exclusivement de ce qui est facile, amusant, curieux, bizarre, passé, sans danger parce qu'il s'agit de sociétés mortes ou lointaines des 
nôtres. Il veut des études concluantes quant au présent. A cette requête on pourrait être tenté de répondre : que la science est souveraine; que sa fantaisie - celle des savants - doit être sans limites. Car on ne sait jamais quel est le fait décisif, même au point de vue pratique. Souvent un fait de nos civilisations a son explication dans d'étranges coins du passé ou de l'exotique. Il est peut-être enregistré en ce moment dans d'obscures statistiques; il peut naître de nos jours, dans des gestations inconnues de formes inconnues d'associations inventées dans des couches inconnues même de nos populations. Ceci s'est vu : la coopération est née ainsi; le syndicalisme a des origines populaires très basses; le christianisme a vécu dans les catacombes; des traditions scientifiques et philosophiques grandioses ont cheminé dans l'obscurité. Mais, ce droit de la science réservé, il faut faire des efforts. - Il faut d'abord être à l'affût de ces mouvements nouveaux des sociétés, les porter au plus vite à la connaissance du public scientifique, en esquisser la théorie. Pour ce faire, il faudrait une meilleure répartition des forces et que, nous-mêmes, nous nous portions davantage vers les choses modernes. L'observation sociologique des institutions d'avenir a un intérêt à la fois théorique et pratique. -Mais ceci ne suffit pas. Le peuple lui-même attend de nous une attitude moins puriste, moins désintéressée. Tout en refusant de sacrifier à une recherche du bien un instant qui ne serait pas exclusivement consacré par la recherche du vrai, il faut évidemment que les sociologues remplissent leur devoir social. Il faut qu'ils aident à diriger l'opinion, voire le gouvernement. Naturellement, si c'est en tant qu'homme politique qu'un sociologue veut agir, il doit, autant qu'il peut, séparer sa science de ses actions. Mais il est possible de produire des travaux sur des sujets moins brûlants, plus généraux et cependant destinés à la pratique morale et à la politique. C'est ainsi que Durkheim concevait sa «morale ». C'est pourquoi nous avons publié sans tarder, même dans la série des Travaux de l'Année sociologique, son Éducation morale, qui ne manque pas de pages politiques; c'est pourquoi nous allons publier encore sa Morale civique et professionnelle. Il y a en effet tout un domaine, à mi-chemin de l'action et de la science, dans la région de la pratique rationnelle où le sociologue doit et peut s'aventurer.

De plus, de temps en temps, par hasard, nous pouvons être sûrs de nos prévisions et même les transformer tout de suite en préceptes. Les savants des heureuses sciences expérimentales, si paisibles et si fiers de leurs méthodes et de leur indépendance, savent, eux, souvent, appliquer leur science à l'industrie ou à la médecine. Ni ils ne craignent la confusion des deux ordres de recherches, ni ils ne redoutent de se rabaisser, ni ils n'ont honte de paraître soit inutiles, soit utiles. De même, il faut imposer notre science comme telle, mais il ne faut pas craindre d'être confondu avec l'homme d'action, quand on le peut, quand on n'a « cherché », comme disait la Bible, et quand on ne parle qu'au nom de la science elle-même. Après avoir fait avancer celle-ci, il faut essayer de l'utiliser. D'ailleurs, sur bon nombre de points, certains des nôtres ont vu clair pratiquement. Les deux Webb en Angleterre, Emmanuel Lévy, à partir de leurs théories du syndicalisme et du contrat, ont beaucoup fait pour instaurer les formes nouvelles du Contrat collectif. Les conclusions du livre de Durkheim sur le Suicide, celles qui concernent le groupe professionnel, devraient être enseignées partout. Sur l'héritage, la leçon de Durkheim ${ }^{1}$ qui est la conclusion de recherches longues et géniales sur la famille mérite d'être classique. $\mathrm{Ne}$ craignons donc pas de verser ces idées et ces faits dans le débat. Nos conclusions pratiques seront rares et de peu d'actualité? Raison de plus pour les répandre libéralement et avec énergie.

Revue philosophique, 1920. 
Le sociologue peut encore être utile à la politique d'une autre façon. Sans se mêler d'elle, ni aux politiciens, ni aux bureaux, il peut aider ceux-ci par des enquêtes impartiales, par le simple enregistrement scientifique de faits, même de ceux dont il ne connaît pas ou ne peut pas tenter la théorie. - le principe que nous énonçons ici n'est pas un rêve pieux; c'est une chose réalisée. Au cours d'un voyage que nous a permis la munificence d'une grande institution scientifique américaine, nous avons pu constater l'importance et la grandeur d'un mouvement de recherches de ce genre aux États-Unis. Puissamment aidés par les particuliers, les États et les villes, les sociologues transforment les informations dont disposent les législateurs, les administrateurs des villes et des grandes institutions. D'abord, États et villes ont, dans leurs bureaux, des départements de recherches. Mais il y a plus. Au lieu de statistiques et de rapports qui ne répondent qu'à des besoins administratifs, on institue des enquêtes complètes, par exemple, sur certaines villes. Celles-ci les confient à des sociologues ou à des statisticiens indépendants, ou bien encore ceux-ci exécutent ces travaux en dehors de toute administration, de leur propre initiative. C'est ce qui se fait par exemple à l'Institut de recherches sociales de Chicago où, avec M. Merriam, collaborent des économistes et des démographes comme M. Marshall ou comme M. Hill et des sociologues comme MM. Park et Burgess. Ailleurs, au lieu de sèches discussions de droit, au lieu des vieilles statistiques criminelles, statistiques des cours et des prisons, non pas de la moralité, on a institué les grandes enquêtes judiciaires détaillées de Cleveland. Celle que $\mathrm{M}$. Pound dirige à Harvard, pour tout l'État de Massachusetts, avec le concours de la Law School de Harvard tout entière, élèves et maîtres, soumet à une analyse précise chacun des cas qui se sont présentés devant les tribunaux; et, ensuite, la «tabulation » de ces cas donne l'état précis de la jurisprudence, celui de la moralité publique et celui des tendances de l'une et de l'autre. Les législateurs, les hommes de loi, les opinions publiques souveraines sont ainsi impartialement renseignés sur eux-mêmes. Les Instituts de recherches économiques sont aussi nombreux là-bas. (Ils commencent à prospérer en Allemagne.) Dans un État, la Caroline du Nord, le Département de science sociale de l'université est chargé des enquêtes législatives préalables à la préparation des lois. Ce mouvement n'est qu'à son début.

En tout état de cause, le sociologue est qualifié professionnellement aussi bien et mieux que les bureaucrates, pour observer même les phénomènes que ceux-ci administrent : car les fonctionnaires n'ont pas naturellement l'impartialité nécessaire et la vue claire des choses; ils représentent surtout la tradition quand ce ne sont pas leurs intérêts à eux ou ceux d'une classe qu'ils servent.

Hors de cela que pouvons-nous faire encore? Bien peu. Mais ce serait déjà bien. Quelques-uns d'entre nous pourraient étudier, pratiquement et théoriquement à la fois, les idées nouvelles et anciennes, les usages traditionnels et les nouveautés révolutionnaires des sociétés qui, en ces moments troublés, cherchent à enfanter leur propre avenir. Si quelques jeunes gens, épris de grandes entreprises, savaient faire cela, les données politiques de notre temps et de chaque société, faits et idéaux, pourraient être étudiés à part et sans préjugés. Les choses présentes pourraient alors faire l'objet d'une sorte de comptabilité intellectuelle, de constante " appréciation » comme disait Comte. Le premier temps d'une politique positive c'est : de savoir et dire aux sociétés en général et à chacune en particulier, ce qu'elles font, où elles vont. Et le second temps de la morale et de la politique proprement dites consiste à leur dire franchement si elles font bien, pratiquement et idéalement, de continuer à aller dans telle ou telle direction. Le jour où, à côté des sociologues, quelques théoriciens de la 
politique ou quelques sociologues eux-mêmes, épris du futur, arriveront à cette fermeté dans le diagnostic et à une certaine sûreté dans la thérapeutique, dans la propédeutique, dans la pédagogie surtout, ce jour-là la cause de la sociologie sera gagnée. L'utilité de la sociologie s'imposera; elle imprimera une formation expérimentale à l'esprit moral et à l'éducation politique; elle sera justifiée en fait, comme elle l'est en raison.

Le principal but sera ainsi atteint le jour où, séparée d'elle, mais inspirée d'elle, une politique positive pourra venir en application d'une sociologie complète et concrète. Si elle ne donne pas les solutions pratiques, elle donnera du moins le sens de l'action rationnelle. L'instruction, l'information, l'entraînement sociologiques donneront aux générations qui montent le sentiment de la délicatesse des procédés de la politique. Ceux-ci, inconsciemment usités en ce moment, pourront être portés au degré de conscience voulue quand une, deux générations de savants auront analysé les mécanismes des sociétés vivantes, celles qui nous intéressent pratiquement. Les hommes politiques et les hommes d'action, ne se borneront plus à des choix instinctifs. Sans attendre une théorie trop poussée, ils sauront consciemment balancer les intérêts et les droits, le passé et le futur. Ils sauront de façon constante estimer ce milieu interne qu'est la société, ces milieux secondaires que forment les générations, les sexes et les sous-groupes sociaux. Ils sauront peser les forces que sont les idées et les idéaux, les courants et les traditions. Ils sauront enfin ne pas méconnaître les milieux externes où se meuvent les intérêts qu'ils administrent : les autres sociétés qui peuvent les contrarier; le sol dont il faut administrer les réserves en vue des générations futures. Voilà, sans utopie, mais sans confusion avec la science, un programme de politique positive.

On trouvera peut-être ce programme bien petit. Ces conclusions sont peut-être décevantes pour l'homme politique, ou même pour le social worker, pour le zélateur $\mathrm{du}$ « service social », pour les auteurs de civics, qui viennent en ce moment s'ajouter généreusement et sans doute efficacement à eux. Mais, une fois le branle donné, il est possible que d'autres effets suivent. Bien des problèmes dont on cherche la solution de front, sont mal posés; d'autres bien posés sont mal traités. La part de l'éducation n'est faite par personne et pourtant elle est peut-être la plus importante de toutes. Le rôle des partis est grandement exagéré par les historiens, par la presse et par l'opinion; la prépondérance des intérêts, surtout de ceux des sous-groupes, économiques en particulier, est vraiment trop grande en ce moment. Au contraire, la part de la morale, spécialement de celle des sous-groupes, par exemple du groupe professionnel, est sous-estimée. Voilà bien des problèmes, des problèmes essentiels que posent les sociologues, mais que lie se posent même pas encore le public, le Parlement, les bureaux. Au contraire ceux-ci nous imposeraient volontiers leurs problèmes à eux, moins importants. Il se peut que la sociologie ne contente ni les corps souverains, ni les sections diverses de nos sociétés.

Il se peut même que, tout en étant utile, elle ne contente personne. La sociologie n'est que le moyen principal d'éducation de la société. Elle n'est pas le moyen de rendre les hommes heureux. Même l'art social et la politique en sont incapables quoiqu'ils poursuivent ce but illusoire. Durkheim l'a bien montré. Science et art n'ont pour effet que de rendre l'homme plus fort et plus maître de lui. Les oeuvres de la raison ne peuvent que donner l'instrument aux groupes et aux individus qui les composent; c'est à ceux-ci qu'il incombe de s'en servir pour leur bien..., s'ils veulent..., s'ils peuvent. 
La sociologie n'a pas de panacée? Ce n'est pas une raison pour arrêter ses progrès. Bien au contraire, il s'agit de la rendre utile en en multipliant les travaux et les étudiants.

Nous ne nous sommes attardé sur ces questions de méthode que parce qu'il s'agit précisément, tout de suite, en un moment où nos études sont populaires, de chercher à donner aux travailleurs qui y participent, le plan qui leur permettra le meilleur choix de leurs travaux. 